\title{
Clinical Features, Prognosis and Susceptibility Genes of 2 Infants With Rhabdomyosarcoma of the Tongue: Case Report
}

\section{Peiyi Yang}

Beijing Children's Hospital

$\mathrm{Na} \mathrm{Xu}$

Beijing Children's Hospital

Yan Su

Beijing Children's Hospital

Chao Duan

Beijing Children's Hospital

Shengcai Wang

Beijing Children's Hospital

Libing Fu

Beijing Children's Hospital

Tong Yu

Beijing Children's Hospital

\section{Ruolan Guo}

Beijing Children's Hospital

Xiaoli Ma ( $\nabla$ mxl1123@vip.sina.com )

Beijing children's hospital https://orcid.org/0000-0001-7930-4944

\section{Case report}

Keywords: infant, spindle cell rhabdomyosarcoma, tongue, susceptibility gene

Posted Date: March 6th, 2021

DOl: https://doi.org/10.21203/rs.3.rs-285585/v1

License: (c) (i) This work is licensed under a Creative Commons Attribution 4.0 International License.

Read Full License 


\title{
Clinical features, prognosis and susceptibility genes of 2 infants with
}

\section{rhabdomyosarcoma of the tongue: case report}

Peiyi Yang ${ }^{1 \#}, \mathrm{Na} \mathrm{Xu}^{1 \#}$, Yan $\mathrm{Su}^{1}$, Chao Duan${ }^{1}$, Shengcai Wang ${ }^{2}$, Libing $\mathrm{Fu}^{3}$, Tong $\mathrm{Yu}^{4}$, Ruolan Guo ${ }^{5}$ and Xiaoli $\mathrm{Ma}^{1}$

\# Peiyi Yang and $\mathrm{Na} \mathrm{Xu}$ contributed equally to this work.

${ }^{1}$ Department of Medical Oncology, Beijing Children's Hospital, Capital Medical University, National Center of Children's Health, Beijing 100045, China; ${ }^{2}$ Department of Otolaryngology Head and Neck Surgery, Beijing Children's Hospital, Capital Medical University, National Center of Children's Health, Beijing 100045, China; ${ }^{3}$ Department of Pathology, Beijing Children's Hospital, Capital Medical University, National Center of Children's Health, Beijing 100045, China; ${ }^{4}$ Department of Image Center, Beijing Children's Hospital, Capital Medical University, National Center of Children's Health, Beijing 100045, China; ${ }^{5}$ Medical Genetics Center of Beijing Pediatric Research Institute, Beijing Children's Hospital, Capital Medical University, National Center of Children's Health, Beijing 100045, China

Corresponding author: Xiaoli Ma, Department of Medical Oncology, Beijing Children's Hospital, Capital Medical University, National Center of Children's Health, Beijing 100045, China Email: mxl1123@vip.sina.com

\begin{abstract}
Background: Rhabdomyosarcoma(RMS) is the most common soft tissue tumor in children. RMS of the oral cavity accounts for $10-12 \%$ of all head and neck RMS, and the tongue is a rare primary site. The most common pathological types of RMS include embryonal rhabdomyosarcoma and alveolar rhabdomyosarcoma, and spindle cell rhabdomyosarcoma(SRMS) is rare. Meanwhile, it has been reported in the literature that infant is a rare onset age in RMS. We review two cases of infant tongue SRMS, and analyze the clinical features, prognosis and susceptibility genes.
\end{abstract}

Case presentation: The clinical data of 2 infants with RMS of the tongue admitted to Beijing Children's Hospital were retrospectively collected. Both the children and their parents accepted exome-customized capture sequencing of single gene disease in peripheral venous blood, and the results were analyzed. Both cases were infants and 
diagnosed with lingual RMS at 3 and 5 months after birth, respectively. The pathological type were both spindle cell rhabdomyosarcoma. The maximum diameter of the tumor in case 1 was $8 \mathrm{~mm}$, and $23 \mathrm{~mm}$ in case 2 . The clinical TNM staging both were TlaN0M0. IRS staging of case 1 was II, and case 2 was I. They were both classified in the low risk group, and treated with surgery and chemotherapy. The regimen of chemotherapy including 4 courses of VAC(vincristine, actinomycin D, cyclophosphamide) regimen first, followed by 4 courses of VA(vincristine, actinomycin D) regimen. Case 1 was in complete remission at the latest follow-up, and case 2 suffered from relapse 10 months after stopping chemotherapy, and achieved complete remission after the multi-disciplinary treatment of chemotherapy, surgery and radiotherapy. The venous blood gene test of the two children did not find pathogenic mutation or possible pathogenic mutation related to RMS. In case 1, variants of $C D K 4$ and $B R C A 1$ gene, both unclear significance (possibly) related with RMS, were detected. For case 2, we found three variants of unknown significance that were (possibly) associated with RMS, including variants of TRIP13, APC, and RAD54L gene.

Conclusions: Infant tongue RMS is rare, the clinical manifestations of which lack specificity, and early recognition is difficult. The success and timing of local treatment are important prognostic factors. Genetic testing may be helpful for early detection of tumor susceptibility and prognosis estimation.

Key words: infant, spindle cell rhabdomyosarcoma, tongue, susceptibility gene

\section{Background}

Rhabdomyosarcoma is the most common soft tissue tumor in children, accounting for about $6.5 \%$ of childhood tumors. The most common primary sites were head, neck and genitourinary system, followed by limbs, trunk and others ${ }^{[1]}$. RMS of the oral cavity accounts for $10-12 \%$ of all head and neck $\operatorname{RMS}^{[2]}$. The tongue, palate and cheek are the most common sites in the oral cavity, among which the tongue is relatively rare $^{[3]}$. The most common pathological types of RMS include embryonal rhabdomyosarcoma and alveolar rhabdomyosarcoma ${ }^{[8]}$, and SRMS is rare, which is now incorporated with sclerosing rhabdomyosarcoma(ScRMS) as an independent subtype of $\mathrm{RMS}^{[4]}$, accounting for about $5 \%$ to $10 \%$ of all the pathological types ${ }^{[5]}$. Meanwhile, it has been 
reported in the literature that infant is a rare onset age in $\mathrm{RMS}^{[6]}$. The median age of 213 children with RMS reported by Beijing Children's Hospital was 48 months, and infants accounted for $7.0 \%{ }^{[7]}$. We report two cases of SRMS originating from the tongue. Analyzing the clinical features of the cases, and exploring the susceptibility genes possibly associated with the disease, may help us improve the understanding of infant SRMS of the tongue.

\section{Case presentation}

\section{Case 1}

A 4-month-old girl was admitted to our hospital with a mass of the tongue, which had been resected already. The mass was discovered on the left lingual margin of the tongue more than 1 month after birth, 3-4 mm in diameter. She wasn't taken to a doctor at that time, and the tumor gradually increased to about $6-8 \mathrm{~mm}$ in diameter 2 months later. Then she was admitted to a local hospital, and was preliminarily diagnosed as "mucoceles on the left margin of the tongue", treated by a surgical resection. The pathologic diagnosis was highly differentiated spindle cell rhabdomyosarcoma. The neoplastic cells are short and fusiform, with atypia and mitosis, and grow infiltratively into the surrounding muscle tissue. The immunohistochemical results showed Desmin(+), Myogenin(+), MyoD1(+), Ki-67(10\%), Myoglobin(+), CD34(+), S-100(-), SMA(-), TFE3(-).

There were no abnormalities in the birth history, maternal pregnancy history or family history of the child.

The enhanced magnetic resonance imaging(MRI) indicated that the soft tissue in the right palatal and parapharyngeal space was slightly thicker than that in the contralateral space, and the strengthening was slightly more obvious. Imaging examination of lung, abdomen and head, bone marrow smear, and bone scan showed no metastatic lesions. The child was finally diagnosed as spindle cell rhabdomyosarcoma of the lingual surface, the clinical TNM staging was T1aN0M0, and the IRS staging was II, classified in the low risk group.

The child started receiving chemotherapy once she was diagnosed at our hospital (chemotherapy began more than 1 month after surgery), including 4 courses of VAC 
regimen first, followed by 4 courses of VA regimen. Then chemotherapy was stopped, and she didn't receive radiotherapy. At her last review, she had been followed up for 26 months, and the tumor was in complete remission without metastasis. The child had normal height, weight and language development, and could eat normally without abnormal taste.

\section{Case 2}

Case 2 was a 3-month-old girl admitted to our hospital with 2 months' duration of choking. A mass on the root of the tongue was found in physical examination(Figure 1) in the local hospital once the symptom appears, and MRI revealed a mass in size of $23 \mathrm{~mm} \times 17 \mathrm{~mm} \times 18 \mathrm{~mm}$. She was preliminarily diagnosed as "tumor of the root of tongue of unknown nature", and was also treated by a surgical resection. Histological examination showed mesenchymal spindle cell tumor, tending to fetal rhabdomyoma. He was admitted to our hospital 2 months after the operation. Pathological consultation in our hospital revealed spindle cell rhabdomyosarcoma. The neoplastic cells were composed of spindle cells arranged in bundles or sheets, with mild atypia. Mitotic images were visible and the neoplastic cells showed infiltrative growth. The immunohistochemical results showed Desmin(+), Myogenin(Scattered+), MyoD1(+), $\operatorname{SMA}(+)$, CD117(-). Fluorescence in situ hybridization(FISH) of the NCOA2 gene related translocation was negative.

There were no abnormalities in the birth history, maternal pregnancy history or family history of the child.

The maxillofacial enhanced MRI of our hospital indicated that the base of the tongue was thick, and lamellar uneven enhancement of fat-saturated T2 signal was observed, which was considered as postoperative change. Imaging examination of lung, abdomen and head, bone marrow smear, and bone scan showed no metastatic lesions. The child was finally diagnosed as spindle cell rhabdomyosarcoma of the tongue, the clinical TNM staging was T1aN0M0, and the IRS staging was I, classified in the low risk group.

The patient started receiving chemotherapy once she was diagnosed definitely (chemotherapy began 2 months after surgery), also including 4 courses of VAC regimen 
followed by 4 courses of VA regimen. She didn't receive radiotherapy either. The maxillofacial enhanced MRI 10 months after the end of the chemotherapy above indicated that the range of nodular and patchy fat-saturated T2 signal in the tongue body and base was larger than before, showing uneven and obvious enhancement, with unclear boundary, involving the geniohyoid muscle, left geniohyoid muscle and mylohyoid muscle(Figure 2). Bilateral cervical lymph nodes were partially enlarged with enhancement. After multidisciplinary consultation, it was concluded that the tumor recurred. Then she received chemotherapy again, which was alternately composed of VDC (vincristine, doxorubicin, cyclophosphamide) regimen and IE (icyclophosphamide, etoposide) regimen, with a total of 11 courses. After the 11 courses ended, surgery was performed and the tumor was resected totally. Postoperative pathology showed no residual tumor cells. Then she accepted chemotherapy of vincristine and irinotecan for 2 courses, followed by proton radiation therapy, with a total dose of 41.4Gy, administered by 23 times. After 31 months of follow-up, the tumor was in completely remission without metastasis. Fortunately, the patient also had normal height, weight and language development, and could eat normally without abnormal taste at the last follow-up.

\section{Single gene disease exome-custom capture sequencing}

With the informed consent of the children's parents, exome-custom capture sequencing of single gene disease was performed. Target sequences were captured, sequenced and analyzed in the exon coding region of more than 6,100 genes known to be associated with human diseases, as well as the $5 \mathrm{bp}$ region of adjacent introns (excluding the untranslated region). Peripheral venous blood of the children and their parents was collected, and genomic deoxyribonucleic acid was extracted for classification of variant pathogenicity. The classification of variation was as follows: pathogenic variation, possible pathogenic variation, unknown significance variation, possible benign variation and benign variation.

The results showed that no pathogenetic variation or possible pathogenetic variation which was consistent with the genealogical inheritance pattern and related to the phenotype of the children was detected in the two cases. In case 1, two variants of 
unclear significance (possibly) related with the disease were detected: (1) heterozygous variation of $C D K 4$ gene (NM000075.3), named C. 806C > T (p.Ala269VAL), from mother, not included in Clinvar/HGMD database, and the software forecasts are inconsistent; (2) Heterozygous mutation of BRCA1 gene (NM007294.3), mutation name was C. 3350T > C (p.Val1117ala), from father, not included in Clinvar/HGDD database, and software predicted that it may be benign. In case 2 , three variants of unknown significance were detected that were (possibly) associated with the disease: (1) Heterozygous variation of TRIP13 gene (NM004237.3), named C. 572C > T (p.Ala191Val), from mother, not included in Clinvar /HGMD database, and predicted by software as deleterious; (2) Heterozygous variation of $A P C$ gene (NM000038.5), named as C. 8437A > G (p.THR2813ALA), from mother, not included in Clinvar /HGMD database, and software prediction is inconsistent. (3) Heterozygous variation of RAD54L gene (NM003579.3), mutation named C. 689T > C (p.Val230Ala), from father, not included in Clinvar /HGMD database, and software predicted as deleterious. In case 2 , three variants (possibly) associated with the disease were also detected, two $R A B 3 G A P 2$ gene variants and one $M M A C H C$ gene variant.

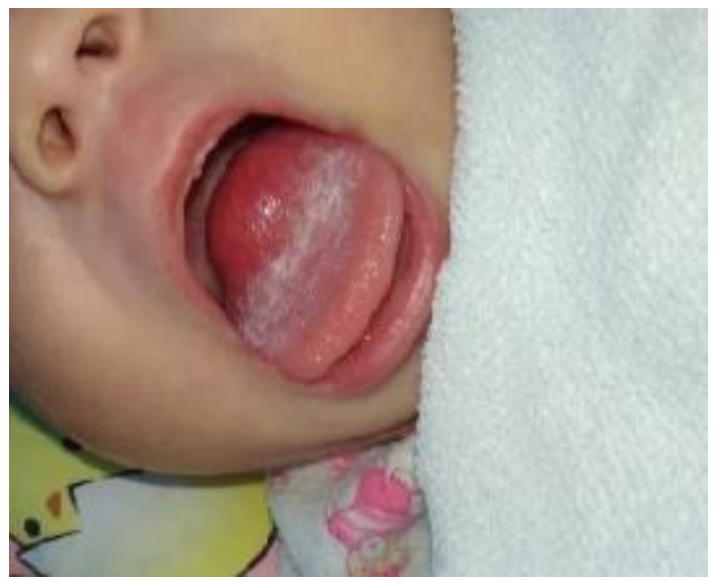

Figure 1 Case 2, tongue appearance at diagnosis 


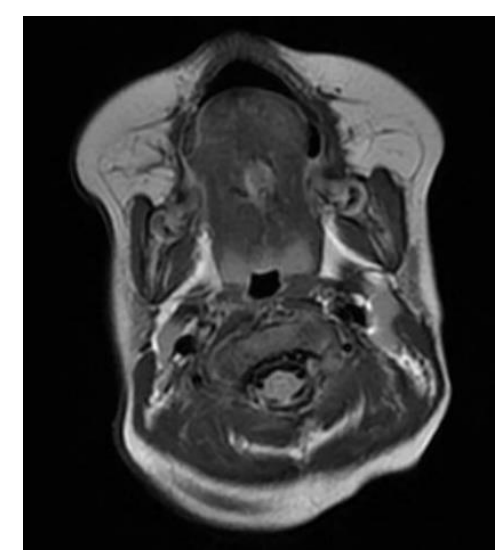

Figure 2 Case 2, tumor in magnetic resonance imaging at relapse

\section{Discussion and Conclusions}

The clinical manifestations of tongue RMS are lack of specificity, and early recognition is difficult, which may lead to misdiagnosis. In early stage of the disease, the tumor expands and infiltrates the muscle from which it arises, presenting first as a well demarcated nodule or polypoid lesion with a soft or gummy consistency. When these lesions grow rapidly they may cause dyspnea, dysphagia, and cough, including acute respiratory obstruction ${ }^{[8]}$. In case 2 , tongue mass was found because of the symptom of choking milk. The differential diagnosis of the mass of tongue includes hemangiomas, fibroma, rhabdomyoma, lymphangioma, and lingual thyroid ${ }^{[8,9]}$, and primary lingual RMS is rare, the definite diagnosis of which requires pathological examination. In the Intergroup Rhabdomyosarcoma Studies(IRS) I, II and III, only 7 rhabdomyosarcomas of the tongue were registered, representing $0.34 \%$ of IRS participants ${ }^{[10]}$. Rejin Kebudi et al. summarized a total of 13 cases of lingual RMS reported in English literatures from 1973 to 2009, whose median age was 3.5 months, much younger than the median age of 5 years in children with RMS reported in literature $^{[9]}$. Our 2 cases were initially considered as mucoceles and tongue root tumors of unknown nature in local hospital respectively. If the possibility of malignant tumors such as RMS were taken into account, and further diagnosis and treatment after surgical resection were performed in time, the optimal timing of treatment may be better grasped. 
Age is one of the risk factors affecting the prognosis of RMS. Previous studies have shown that age is a strong, independent risk factor, even after accounting for other factors such as group, stage, and tumor histology. Infants younger than 1 year and adolescents older than 10 years diagnosed with RMS had poorer survival than did children aged $1-9^{[11]}$. Genetic differences in tumors, compliance with therapy, tolerance of therapy, differences in drug metabolism, or different levels of drug resistance within tumors may explain how age affects prognosis. The most recognized reason now is that taking into account the complications and long-term adverse effects caused by surgery and radiotherapy, the local treatment intensity of infants is often low, leading to poor treatment outcome ${ }^{[12]}$. Among the 13 cases of lingual RMS reviewed by Rejin Kebudi et al, one 10-month-old girl received surgery after chemotherapy, the surgical margin was positive, and relapsed after 30 months of follow-up ${ }^{[9]}$.

It was previously thought that young children couldn't tolerate radiation therapy, but it is now believed that when radiation is omitted, even in those with Stage I disease, there is a high risk of recurrence, with local recurrence being the most common, confirming the need for radiation therapy. Therefore, brachytherapy, proton therapy, intensity modulated radiation therapy and other emerging precision radiotherapy methods may improve both the prognosis of infant and radiotherapy tolerance ${ }^{[13]}$. Case 2 was a patient of IRS Stage I, who did not receive radiotherapy and had local recurrence 10 months later, received proton therapy after recurrence. Her condition was well controlled at present. Van Grotel et al. reported in 2003 that surgical debulking of the tumor combined with consecutive brachytherapy was used to treat a child who was resistant to chemotherapy, and the tumor was completely remission after 3 years of follow-up, with little impact on eating and language function ${ }^{[14]}$. In addition, attention should be paid to the timing of local and systemic treatment. If the tumor is surgically removed first, chemotherapy should be started within 7 days after surgery ${ }^{[1]}$. Case 2 started chemotherapy 2 months after the first operation, the interval was long, which may also be related to the recurrence. 
SRMS is a rare type of rhabdomyosarcoma, which mainly occurs in the paratesticular region and head and neck of children and adolescents. In the WHO classification of soft tissue and bone tumors (2013 edition), SRMS and ScRMS were combined and classified as an independent subtype of $\mathrm{RMS}^{[4]}$, accounting for about $5 \%$ to $10 \%{ }^{[5]}$. Due to the similarity between SRMS and other spindle cell tumors, accurate diagnosis is very challenging. Therefore, it is of great importance to use immunohistochemistry and even molecular genetic tests to make a clear diagnosis and differential diagnosis of SRMS. MyoD1 was strongly positive in SRMS/ SCRMS, desmin was diffuse, and myogenin was weak ${ }^{[15]}$, and the pathological immunohistochemistry of our two cases was consistent with the characteristics above. Current data supports that certain SRMS/ScRMS have a more aggressive clinical course with reduction of long-term survival, and those found in the head and neck region often exhibit extensive local recurrence ${ }^{[16]}$. Due to its genetic heterogeneity, its prognosis varies with different molecular biological characteristics. Patients younger than 1 year old who involved $V G L L 2, S B F, T E A D 1$ and NCOA2 gene fusion had better prognosis, while patients with MYOD1 gene mutation showed stronger aggression and higher mortality, regardless of age. Given that this distinct molecular subtype is characterized by an aggressive biologic behavior compared to other genetic subtypes of spindle and sclerosing rhabdomyosarcoma, the MYODI genotype has been recommended to be used as a molecular marker in both subclassification and prognostication of SRMS/ScRMS ${ }^{[17]}$. The two cases in this report were both SRMS in infant, and the therapeutic effect were different. That may be related to the different molecular biological characteristics. In case 2, there was no $N C O A 2$ gene-related translocation detected by FISH, but she didn't get the examination of MYOD1 gene mutation, so it was difficult to judge the consistency between the gene variation above and prognosis. For patients with SRMS/ ScRMS, the above gene tests should be performed as far as possible to improve the accuracy of prognosis judgment and achieve stratified treatment.

It is also important to note that infantile onset RMS may be associated with a potential cancer predisposition syndrome $(\mathrm{CPS})^{[18]}$. It has been reported in domestic 
and foreign literatures that the occurrence and development of CPS-related RMS are related to different genetic characteristics (such as chromosomal translocation, heterozygosity deletion, etc.) and molecular pathway changes (such as some tumor suppressor genes and oncogenes) ${ }^{[19]}$, which may cause chemotherapy intolerance or no response, and some patients need long-term follow-up before diagnosis. The family syndromes associated with RMS include Li-Fraumeni syndrome (LFS), neurofibromatosis type 1, Rubinstein-Taybi syndrome, Beckwith-Wiedemann syndrome, and Costello syndrome, etc ${ }^{[20]}$. The pedigree of the lingual RMS patient reported by Rejin Kebudi et al above conformed to the characteristics of LFS (the proband was a 2-year-old boy with RMS, his aunt suffered from premenopausal breast cancer, and his uncle under 60 years of age was diagnosed as prostate carcinoma) ${ }^{[9]}$. RMS is the most common pediatric malignancy in the typical LFS family ${ }^{[7]}$, and the occurrence of LFS is related to the germ line mutation of the tumor suppressor gene $T P 53^{[20]}$. In addition, in the case of tongue RMS reported by Van Grotel et al above, after 15 years of follow-up, there were multiple skin lesions on the patient's right shoulder and back, and the biopsy pathology was basal cell carcinoma. Due to the presence of mandibular bone cyst at the same time, genetic testing was performed and PTCH1 gene mutation was confirmed, thus Gorlin syndrome was diagnosed ${ }^{[21]}$. It is suggested that some genetic susceptibility syndrome can only be detected after longterm follow-up in some cases according to the accompanying phenotype, so it is necessary to conduct long-term monitoring.

Both of the two cases were diagnosed with RMS in infancy, so they accepted exome-custom capture sequencing of single gene disease of the peripheral blood to find out whether they had the gene variation currently known to be associated with RMS. Among the mutations found in the test that may be associated with RMS, $C D K 4$ gene (case 1) was located at 12q14, encoding the cell cycle regulator CDK4, and its amplification was observed in ARMS, breast cancer, non-small cell lung cancer, glioblastoma multiforme, etc ${ }^{[22]}$. BRCA1 gene (case 1), located at $17 \mathrm{q} 21$, is a tumor suppressor gene, and its mutation can be seen in breast cancer, ovarian cancer, etc $^{[23]}$. TRIP13 gene (case 2) is located in 5p15 and can be highly expressed in a 
variety of malignant tumors (but no RMS has been reported) ${ }^{[24]}$. APC gene (case 2), located at $5 \mathrm{q} 21-22$, is also a tumor suppressor gene, and its inactivation or mutation is associated with colorectal cancer, myeloma, RMS, adenocarcinoma, etc ${ }^{[25]} \cdot R A D 54 L$ gene (case 2) was located at $1 \mathrm{p} 32$, and its mutation may be associated with breast cancer, colon cancer, lymphoma, meningioma, etc ${ }^{[26]}$. The mutations in the genes mentioned above in the two children were classified as unknown mutations that were not included in Clinvar /HGMD database. Although it cannot be concluded that the occurrence of RMS in the two cases is related to congenital genetic factors, in consideration of their onset age, primary site and pathological type, it is necessary to continue long-term follow-up to observe the outcome of the disease and summarize other cases meanwhile to reach a conclusion.

This study reported two cases of infant tongue RMS, and analyze their clinical features, prognosis and susceptibility genes. Infant tongue RMS is rare, its clinical manifestations lack specificity, and early recognition is difficult. The success and timing of local treatment are important prognostic factors. Genetic testing may be beneficial to early detection of tumor susceptibility and prognosis, and provide a theoretical basis for long-term follow-up of patients and their families.

\section{List of abbreviations}

RMS: Rhabdomyosarcoma

SRMS: spindle cell rhabdomyosarcoma

VAC: vincristine, actinomycin D, cyclophosphamide

VA: vincristine, actinomycin D

ScRMS: sclerosing rhabdomyosarcoma

MRI: magnetic resonance imaging

FISH: fluorescence in situ hybridization

VDC: vincristine, doxorubicin, cyclophosphamide

IE: icyclophosphamide, etoposide

IRS: Intergroup Rhabdomyosarcoma Studies

CPS: cancer predisposition syndrome

LFS: Li-Fraumeni syndrome 


\section{Ethics approval and consent to participate}

Patients and their parents provided written informed consent for the study. This case report was approved by ethics committees of the Ethics Committee of Beijing Children's Hospital, Capital Medical University(2018-k-106).

\section{Consent for publication}

Written informed consents were obtained from the parents of the patients for publication of this case report, all information contained within it and any accompanying images. Copies of the written consents are available for review by the editor of this journal.

\section{Availability of data and materials}

All data generated or analyzed during this study are included in this published article.

\section{Competing interests}

The authors declare that they have no competing interests.

\section{Funding}

This work was supported by the Pediatric Medical Coordinated Development Center of Beijing Hospitals Authority (XTYB201803). The funders had no role in the study design, data collection and analysis, decision to publish, or preparation of the manuscript.

\section{Authors' contributions}

XLM designed the idea for this article and had managed the cases. NX collected clinical data. PYY and YS analyzed and interpreted the patients' data. LBF performed the histological examination. TY analyzed the MRI images. RLG performed the single gene disease exome-custom capture sequencing. PYY, NX and XLM were major contributors in writing the manuscript. All authors were involved in drafting, reviewing and approved the final version for submission.

\section{Acknowledgements}

All authors would like to thank the patients and their parents.

\section{Author information}

Department of Medical Oncology, Beijing Children's Hospital, Capital Medical University, National Center for Children's Health, Beijing, China 
Peiyi Yang, Na Xu, Yan Su, Chao Duan \& Xiaoli Ma

Department of Otolaryngology Head and Neck Surgery, Beijing Children's Hospital, Capital Medical University, National Center for Children's Health, Beijing, China Shengcai Wang

Department of Pathology, Beijing Children's Hospital, Capital Medical University, National Center for Children's Health, Beijing, China

Libing Fu

Department of Image Center, Beijing Children's Hospital, Capital Medical University, National Center for Children's Health, Beijing, China

Tong $\mathrm{Yu}$

Medical Genetics Center of Beijing Pediatric Research Institute, Beijing Children's Hospital, Capital Medical University, National Center for Children's Health, Beijing, China

Ruolan Guo

\section{References}

1. Ni X, Ma XL, Wang HM, et al.

http://www.nhc.gov.cn/yzygj/s3593/201909/5f1d3329606e4cd2aa6e501603703ee4/fil es/8436355a7b054d9c9c9f1834bf32f781.pdf. Accessed 28 February 2021.

2. R Chigurupati, A Alfatooni, et al. Orofacial rhabdomyosarcoma in neonates and young children: a review of literature and management of four cases. Oral oncology. 2002;38(5):508-515. doi:10.1016/s1368-8375(01)00087-2.

3. Antonietta Cirocco, Francisco González, Ana María Sáenz, et al. Embryonal Rhabdomyosarcoma of the Tongue. Pediatric Dermatology. 2005;22(3): 218-221.doi: 10.1111/j.1525-1470.2005.22308.x.

4. Wang J, Zhu XZ. Interpretation of 2013 World Health Organization classification of soft tissue and bone tumours. Chin J Pathol. 2013;42(6):363-365. doi:

10.3760/cma.j.issn.0529-5807.2013.06.002.

5. Yasui N, Yoshida A, Kawamoto H, et al. Clinicopathologic analysis of spindle cell/sclerosing rhabdomyosarcoma. Pediatr Blood Cancer. 2015;62(6):1011-1016. doi:10.1002/pbc. 25367. 
6. Suman Malempati, David A Rodeberg, et al. Rhabdomyosarcoma in infants younger than 1 year: a report from the Children's Oncology Group.Cancer 2011 Aug 01;117(15):3493-501. doi:10.1002/cncr.25887.

7. Xu N, Duan C, Jin M, et al. Clinical and prognostic analysis of single-center multidisciplinary treatment for rhabdomyosarcoma in children. Chin J Pediatr. 2019;57(10):767-773. doi: 10.3760/cma.j.issn.0578-1310.2019.10.008.

8. Antonietta Cirocco, Francisco González, et al. Embryonal rhabdomyosarcoma of the tongue. Pediatric dermatology. 2005 May-Jun;22(3):218-221. doi:10.1111/j.15251470.2005.22308.x.

9. Rejin Kebudi, Gül Nihal Ozdemir. Rhabdomyosarcoma of the tongue: report of a case and review of the literature. Pediatric hematology and oncology. 2011;28(1):6064. doi:10.3109/08880011003749678.

10. Shannon M Kraft, Vivekanand Singh, et al. Differentiating between congenital rhabdomyosarcoma versus fibromatosis of the pediatric tongue.International journal of pediatric otorhinolaryngology. $2010 \mathrm{Jul} ; 74(7): 781-785$.

doi:10.1016/j.ijporl.2010.03.057.

11. D Joshi, James R Anderson, et al. Age is an independent prognostic factor in rhabdomyosarcoma: a report from the Soft Tissue Sarcoma Committee of the Children's Oncology Group.Pediatric blood \& cancer. 2004 Jan;42(1):64-73. doi:10.1002/pbc.10441.

12. Julie A Bradley, Mark L Kayton, et al. Treatment Approach and Outcomes in Infants With Localized Rhabdomyosarcoma: A Report From the Soft Tissue Sarcoma Committee of the Children's Oncology Group. International journal of radiation oncology, biology, physics. 201901 01;103(1):19-27. doi:10.1016/j.ijrobp.2018.08.017.

13. Dev R Puri, Leonard H Wexler, et al. The challenging role of radiation therapy for very young children with rhabdomyosarcoma. International journal of radiation oncology, biology, physics. 2006 Jul 15;65(4):1177-1184.

doi:10.1016/j.ijrobp.2006.02.014.

14. M van Grotel, P Nowak, et al. Long-term remission after non-radical surgery combined with brachytherapy in an infant with a chemo-resistant rhabdomyosarcoma of the tongue. Medical and pediatric oncology. 2003 Dec;41(6):558-561. doi:10.1002/mpo.10431. 
15. Rekhi B, Upadhyay P, Ramteke MP, et al. MYOD1(L122R) mutations are associated with spindle cell and sclerosing rhabdomyosarcoma with aggressive clinical outcomes. ModPathol. 2016; 29(12):1532-1540.

16. Molly Housley Smith, Daniel Atherton, et al. Rhabdomyosarcoma, Spindle Cell/Sclerosing Variant: A Clinical and Histopathological Examination of this Rare Variant with Three New Cases from the Oral Cavity. Head and neck pathology. 2017;11(4):494-500. doi:10.1007/s12105-017-0818-х.

17. Narasimhan P Agaram, Michael P LaQuaglia, et al. MYOD1-mutant spindle cell and sclerosing rhabdomyosarcoma: an aggressive subtype irrespective of age. A reappraisal for molecular classification and risk stratification. Modern pathology, 2019;32(1):27-36. doi:10.1038/s41379-018-0120-9.

18. Julia Taeubner, Triantafyllia Brozou, et al. Congenital embryonal rhabdomyosarcoma caused by heterozygous concomitant PTCH1 and PTCH2 germline mutations. European journal of human genetics. 2018;26(1):137-142. doi:10.1038/s41431-017-0048-4.

19. Li DL, Liu CX, Zou H. et al. Advances in molecular biology of rhabdomyosarcoma. Chin J Clin Exp Pathol. 2011;27(2):181-185. doi: 10.3969/j.issn.1001-7399.2011.02.018.

20. Daniela Egas-Bejar, Winston W Huh. Rhabdomyosarcoma in adolescent and young adult patients: current perspectives. Adolescent health, medicine and therapeutics. 2014;5:115-125. doi:10.2147/AHMT.S44582.

21. Petra M van Rijswijk, Marry M van den Heuvel-Eibrink, et al. Very Long-term Sequelae After Nonradical Surgery Combined With Brachytherapy in an Infant With a Chemotherapy-resistant Rhabdomyosarcoma of the Tongue.Journal of pediatric hematology/oncology. 2017 10;39(7):566-569.

doi:10.1097/MPH.0000000000000935.

22. Francesco Schettini, Irene De Santo, et al. CDK 4/6 Inhibitors as Single Agent in Advanced Solid Tumors. Frontiers in oncology. 2018;8:608.

doi:10.3389/fonc.2018.00608.

23. Eliot M Rosen, Michael J Pishvaian. Targeting the BRCA1/2 tumor suppressors. Current drug targets. 2014;15(1):17-31. doi:10.2174/1389450114666140106095432. 24. S Lu, J Qian, et al. Insights into a Crucial Role of TRIP13 in Human Cancer. Computational and structural biotechnology journal. 2019;17:854-861. doi:10.1016/j.csbj.2019.06.005. 
25. Z Li, W Li, et al. Cilia, adenomatous polyposis coli and associated diseases. Oncogene. 2012;31(12):1475-83. doi:10.1038/onc.2011.351.

26. Paola E Leone, Marta Mendiola, et al. Implications of a RAD54L polymorphism $(2290 \mathrm{C} / \mathrm{T})$ in human meningiomas as a risk factor and/or a genetic marker. BMC cancer. 2003;3:6. doi:10.1186/1471-2407-3-6. 


\section{Figures}

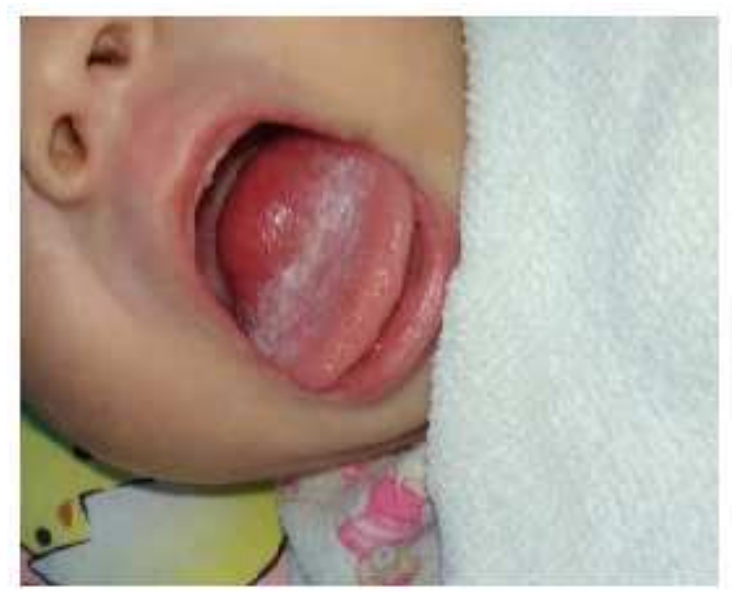

\section{Figure 1}

\section{Case 2, tongue appearance at diagnosis}

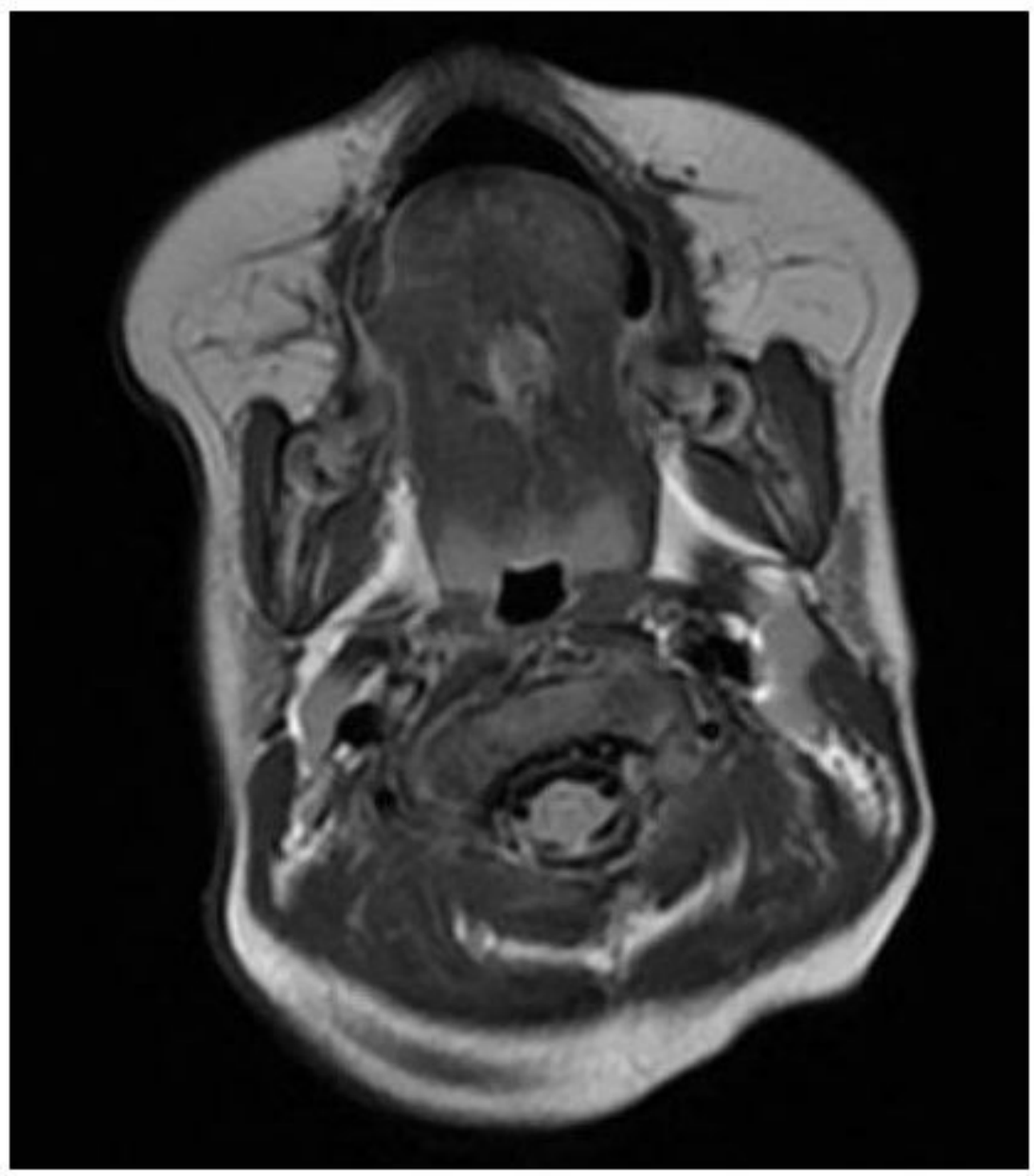

Figure 2

Case 2, tumor in magnetic resonance imaging at relapse 